\title{
Bad for cats, good for humans? Modified feline immunodeficiency virus for gene therapy
}

\begin{abstract}
Ronald G. Crystal
Institute of Genetic Medicine, Weill Medical College of Cornell University, 520 East 70th Street, ST 505, New York, New York 10021, USA. Phone: (212) 746-2258; Fax: (212) 746-8383; E-mail: geneticmedicine@mail.med.cornell.edu.
\end{abstract}

When the concepts of gene therapy were evolving in the late 1980s, the focus was primarily on the hereditary disorders. This was a logical choice for the budding community of gene therapists, in that most hereditary disorders are autosomal recessive deficiency diseases that can theoretically be corrected by transfer of sufficient amounts of the normal gene to the cells manifesting the disease. This concept may be logical, but putting it into practice has been a real challenge. In this issue of the JCI, Wang et al. (1) describe the adaptation of feline immunodeficiency virus (FIV) to transfer the cystic fibrosis (CF) transmembrane conductance regulator (CFTR) CDNA to the respiratory epithelium. This strategy represents a new approach to overcoming some of the challenges in using gene therapy to correct the respiratory manifestations of CF.

Unlike treatment for hematologic disorders, for which target cells for gene therapy can be removed from an individual and subjected to gene transfer manipulations in vitro, gene transfer to internal organs such as the lung requires the gene to be transferred in vivo. In treating the respiratory manifestations of $\mathrm{CF}$, the targets for gene therapy are the $10^{10}$ epithelial cells lining the airways (2). Because of mutations in the 2 parental CFTR genes, the airway epithelial cells of individuals with CF have a deficiency of CFTR function, manifested by decreased $\mathrm{Cl}^{-}$ transport in response to elevations of intracellular cAMP. It is estimated that correction of $5-10 \%$ of airway epithelial cells would be sufficient to prevent the clinical manifestations of CF. The level of normal gene expression required for each cell is not the problem, because the normal airway epithelium has an average of only 1-2 normal CFTR mRNA copies. However, because CF is a hereditary disorder, persistent expression of the newly transferred gene is essential for successful treatment.
To date, most investigators have focused their attention on 3 basic strategies for the transfer the CFTR cDNA to the airway epithelium: use of adenovirus vectors, adeno-associated virus vectors, or plasmids combined with liposomes. All 3 strategies have been evaluated in humans with CF (3, 4-10). The adenovirus vectors are by far the most effective, providing the highest levels of gene expression in the respiratory epithelium of any gene vector system - levels that are sufficient to treat the disease (3). The challenge in the adenovirus strategy is that host defenses limit the persistence of expression $(3,11)$. Adeno-associated virus vectors have a theoretical advantage in that, if they can reach the nucleus of the target cell and be converted from their normal singlestranded form to double-stranded DNA, they may be able to integrate into the host chromosome and thus provide long-term expression (10, 12, 13). Unfortunately, there seems to be a block in the lung epithelium at some step in this process, and these vectors, in their current form, yield clinically inadequate levels of expression. Likewise, the plasmid/liposome combinations are very inefficient, and expression in the lung is very low and only transient $(6,8)$.

From these observations, some rules have evolved that define the modifications to the current vector systems required to successfully treat respiratory manifestations of CF. If adenovirus vectors are to be used, they will have to be more efficient in entering the epithelium (so less vector can be used, thus limiting the extent of the host response to the vector), and/or the vectors will have to be designed to avoid triggering the adaptive and innate host defenses arrayed against the vector, a feature described as "stealth." If adenoassociated virus vectors are to be successful, strategies will have to be developed to bypass biological impediments to integration within the host genome.
Finally, if plasmids combined with liposomes are to be effective, signals will have to be built into the vector system to guide the plasmid into the cytoplasm, and then find, enter, and persist in the nucleus (14).

The study by Wang et al. (1) uses an alternative vector system that seems well-suited to the challenge of transferring and expressing the normal human CFTR cDNA in the differentiated airway epithelium. The strategy is based on FIV, a T-lymphotrophic retrovirus first identified in 1987 in pet cats in Davis, California (see ref. 15 for a review of FIV). Based on its morphologic and biochemical characteristics, cell tropism, $\mathrm{Mg}^{2+}$ dependent reverse transcriptase, genetic organization, and antigenic properties, FIV is classified (along with HIV) as a lentivirus. Among the lentiviruses, FIV is most similar to the Visna maedi virus. FIV is $105-125 \mathrm{~nm}$ in diameter, spherical to ellipsoid in shape, with short, poorly defined projections on its envelope. The 9.4-kb FIV genome is organized like that of other retroviruses, with env, gag, and pol genes, with an additional 7 open reading frames that encode proteins. Wild-type FIV is trophic for $\mathrm{T}$ cells, B cells, macrophages, astrocytes, and microglia. Once it enters the cell, FIV has a life cycle similar to that of other retroviruses. Cats infected with FIV develop acute lymphadenopathy, neutropenia, and fever. As with HIV infection in humans, this is followed by an asymptomatic phase lasting years, after which generalized lymphadenopathy, recurrent fevers, apathy, leukopenia, anemia, anorexia, weight loss, stomatitis, and behavioral problems ensue. Finally, there is a terminal AIDS-like phase, marked by opportunistic infections, neoplasia, and neurologic abnormalities.

By having genes deleted that wildtype FIV requires to direct its own replication, can be converted into a replication-incompetent virus, capable of transferring recombinant cDNAs such 
as CFTR, but incapable of reproducing except in a producer cell line that expresses the deleted genes $(1,16,17)$. The concept of using a retrovirus for gene therapy is not new - the gene therapy field started with the idea of using modified forms of the Moloney murine leukemia virus (MMLV) to create a replication-deficient vector to transfer genes to target cells (14). The problem with using MMLV-based gene transfer vectors for CFTR gene transfer is that lung epithelial cells are slow to replicate, and the MMLV vectors require the target cell to proliferate for the viral genome with its recombinant gene to reach the target cell nucleus. Not so for FIV. For reasons that are not clearly understood, FIV is capable of delivering its genetic cargo to the nucleus of quiescent cells, where it randomly integrates into the host genome (15). For gene therapy for a hereditary disease, this is a clear advantage, because the newly delivered, integrated gene is permanently transferred from parent cells to daughter cells. In contrast, adenovirus vectors deliver their recombinant genomes to an extrachromosomal location, and thus when the target cell does divide, only 1 daughter cell is genetically modified (14).

To take advantage of the ability of FIV to infect slowly replicating human airway cells, another hurdle must be overcome - wild-type FIV does not infect humans. FIV primarily infects the modern domestic cat (Felis catus), and to a lesser extent large wild cats, but not other species (15). There is no evidence to link FIV to any human disease, and humans bitten by infected cats do not develop anti-FIV antibodies. Thus, an FIV gene transfer vector is useless for potential human gene transfer studies unless its coat is modified to interact with receptors on human cells. To circumvent this limitation, Wang et al. (1) modified the FIV vector to be pseudotyped with a vesicular stomatitis virus $G$ (VSV-G) protein coat, a strategy that has been used to alter the host range of other retrovirus gene transfer vectors (16-18).

Does a replication-incompetent, VSV$G$ pseudotyped FIV vector solve the challenges of effectively transfecting human airway epithelial cells? The answer is ... partially. The human airway epithelium is well designed to avoid viral infection, in that it hides most of the receptors that viruses use for entry - including the receptor used by VSV-G on its basolateral epithelial surface (19, 20). Thus, the FIV vector may be capable of inserting its gene into the host genome, but it cannot do this unless it can enter the cell. To overcome this obstacle, Wang et al. (1) transiently opened the tight junctions of the epithelium, using a formulation that was hypotonic and contained the calcium chelator EGTA (21-23). Together, the combination seems effective, with the VSV-G pseudotyped FIV vector capable of delivering sufficient CFTR cDNA to correct the $\mathrm{Cl}^{-}$transport defect of human airway epithelium derived from individuals with CF. Furthermore, the FIV vectors formulated with EGTA transduced $5-10 \%$ of the large airway epithelial cells, a percentage within the range thought to be sufficient to correct the CF defect $(2,3,7)$. Finally, the FIV vector corrected the $\mathrm{CF}$ defect in vitro for the 6-month life of the epithelial cultures, in vivo infection of tracheal epithelium demonstrated transduction of epithelial cells with progenitor capacity, and the in vivo FIV-transduced epithelia persisted for at least 6 weeks.

Has the battle been won? Hardly - it has only been joined. The FIV vector system is a novel approach to the challenge of transferring CFTR cDNA to the airway epithelium, but there are significant hurdles still to overcome. Like other retroviruses, FIV integrates randomly, making its use in gene therapy a theoretically risky strategy since it could be associated with induction of a malignant phenotype, inability to shut off excess expression of the new gene, and variable expression among cells. The use of VSV-G pseudotyping enables the FIV vector to expand its host range to human cells, but the paucity of receptors for VSV-G on the apical surface airway epithelial cells required Wang et al. (1) to loosen the tight junctions of the airway epithelium to allow the vector to reach the basolateral surface, with its richer density of relevant receptors. Moreover, there is insufficient experience with this technique to determine whether host shut-off of the transferred expression cassette (a problem that has plagued other retrovirus gene transfer vectors) will occur with FIV vectors. Another problem that will have to be overcome is that of developing the methodology to produce large amounts of the modified FIV without contamination by wild-type FIV. FIV is an immunodeficiency virus that is fatal to domestic cats; if modified to infect humans, there is a small risk that vectors will be contaminated with a recombinant wild-type FIV that is trophic for human cells. Although this scenario appears very unlikely, and although such a virus would probably not have the same growth potential as true wildtype FIV, FIV infection could be devastating for the individual patient and could pose a risk to the environment.

From studies with adenovirus-based vectors, we now know that it is possible to transfer the normal CFTR cDNA to the airway epithelium of individuals with CF at levels that theoretically could prevent the clinical manifestations of the disease (3). The major challenge for CF gene therapy is to get the newly transferred gene to be expressed in a persistent fashion. The study by Wang and colleagues (1) represents an advance toward this goal.

1. Wang, G., et al. 1999. Feline immunodeficiency virus vectors persistently transduce nondividing airway epithelia and correct the cystic fibrosis defect. J. Clin. Invest. 104:R55-R62

2. Korst, R.J., et al. 1995. Gene therapy for the respiratory manifestations of cystic fibrosis. Am. J. Respir. Crit. Care Med. 151(Suppl.):S75-S87.

3. Harvey, B.-G., et al. 1999. Airway epithelial CFTR mRNA expression in cystic fibrosis patients after repetitive administration of a recombinant adenovirus. J. Clin. Invest. 104:1245-1255.

4. Zabner, J., et al. 1993. Adenovirus-mediated gene transfer transiently corrects the chloride transport defect in nasal epithelia of patients with cystic fibrosis. Cell. 75:207-216.

5. Bellon, G., et al. 1997. Aerosol administration of a recombinant adenovirus expressing CFTR to cystic fibrosis patients: a phase I clinical trial. Hum. Gene Ther. 8:15-25.

6. Caplen, N.J., et al. 1995. Liposome-mediated CFTR gene transfer to the nasal epithelium of patients with cystic fibrosis. Nat. Med. 1:39-46.

7. Crystal, R.G., et al. 1994. Administration of an adenovirus containing the human CFTR cDNA to the respiratory tract of individuals with cystic fibrosis. Nat. Genet. 8:42-51.

8. Gill, D.R., et al. 1997. A placebo-controlled study of liposome-mediated gene transfer to the nasal epithelium of patients with cystic fibrosis. Gene Ther. 4:199-209.

9. Knowles, M.R., et al. 1995. A controlled study of adenoviral-vector-mediated gene transfer in the nasal epithelium of patients with cystic fibrosis. N. Engl. J. Med. 333:823-831.

10. Wagner, J.A., et al. 1998. Efficient and persistent gene transfer of AAV-CFTR in maxillary sinus. Lancet. 351:1702-1703.

11. Wilson, J.M. 1996. Adenoviruses as gene-delivery vehicles. N. Engl. J. Med. 334:1185-1187.

12. Flotte, T., et al. 1996. A phase I study of an adenoassociated virus-CFTR gene vector in adult CF patients with mild lung disease. Hum. Gene Ther. 7:1145-1159.

13. Duan, D., et al. 1998. Circular intermediates of recombinant adeno-associated virus have defined structural characteristics responsible for longterm episomal persistence in muscle tissue. $J$. Virol. 72:8568-8577.

14. Crystal, R.G. 1995. Transfer of genes to humans: 
early lessons and obstacles to success. Science. 270:404-410

15. Hartmann, K. 1999. Feline immunodeficiency virus infection: an overview. Vet. J. 155:123-137.

16. Johnston, J.C., et al. 1999. Minimum requirements for efficient transduction of dividing and nondividing cells by feline immunodeficiency virus vectors. J. Virol. 73:4991-5000.

17. Poeschla, E.M., Wong-Staal, F., and Looney, D.J. 1998. Efficient transduction of nondividing human cells by feline immunodeficiency virus lentiviral vectors. Nat. Med. 4:354-357.

18. Goldman, M.J., Lee, P.S., Yang, J.S., and Wilson, J.M. 1997. Lentiviral vectors for gene therapy of cystic fibrosis. Hum. Gene Ther. 8:2261-2268.

19. Pickles, R.J., et al. 1998. Limited entry of adenovirus vectors into well-differentiated airway epithelium is responsible for inefficient gene transfer. J. Virol. 72:6014-6023.

20. Walters, R.W., et al. 1999. Basolateral localization of fiber receptors limits adenovirus infection from the apical surface of airway epithelia. J. Biol.
Chem. 274:10219-10226.

21. Anderson, J.M., and Van Itallie, C.M. 1995. Tight junctions and the molecular basis for regulation of paracellular permeability. Am. J. Physiol. 269:G467-G475.

22. Denker, B.M., and Nigam, S.K. 1998. Molecular structure and assembly of the tight junction. Am. J. Physiol. 274:F1-F9.

23. Widdicombe, J.H., Azizi, F., Kang, T., and Pittet, J.F. 1996. Transient permeabilization of airway epithelium by mucosal water. J. Appl. Physiol. 81:491-499. 